\title{
AN EQUIVALENT LINEARIZATION FOR THE DRAG FORCE IN THE MORISON'S EQUATION USING HERMITE POLYNOMIAL ERROR SAMPLE FUNCTION
}

\author{
N. H. THINH $(*)$, N. D. ANH $(* *)$, D. A. CUONG $\left(^{*}\right)$ \\ $\left(^{*}\right)$ University of Le Quy Don, Hanoi, Vietnam \\ $\left(^{* *}\right)$ Institute of Mechanics, Hanoi, Vietnam
}

\begin{abstract}
Within the scope Gaussian equivalent linearization (GEL), a new mean square criterion based on Hermite polynomial error sample functions for determining the coefficients of the equivalent linearization is proposed to linearize non-linear functions of the zero mean Gaussian random process. Application to the Morison's equation for wave forces is presented that shows significant improvements over the corresponding accuracy of the classical GEL.
\end{abstract}

\section{Introduction}

There has been a large amount of the extensive investigations into the response of non-linear stochastic systems due to the fact that many excitations of engineering interest are basically random in nature. Since all real engineering systems are, more or less, non-linear, it is necessary to develop approximate techniques to determine the response statistics of non-linear systems under random excitation. One of the known approximate techniques is the Gaussian equivalent linearization (GEL) which was fist proposed by Caughey [1959] and has been developed by many authors, see e.g. [Atalik \& Utku, 1976] [Casciati \& Faravelli, 1986] [Anh \& Schiehlen, 1997] [Roberts \& Spanos, 1990]. It has been shown that GEL is presently the simplest tool widely used for analysis of non-linear stochastic problem, however, the major limitation of this method is seemingly that it's accuracy decreases as the non-linearity increases and it can lead to unacceptable errors in the second moments. Further, if one needs more accurate approximate solutions there is no way to obtain them using the conventional version of GEL.

To obtain a series of approximate solution in this excellent technique a mean square criterion based on Hermite polynomial sample functions is proposed for determining the coefficients of linearization. The criterion is based on the error sample functions chosen in the form of Hermite polynomials. The proposed technique is then applied to Morison's equation for wave forces. It is obtained that the technique yields significant improvement over corresponding accuracy of the 
classical GEL.

\section{Linearization of the drag force in the Morison's equation}

Wave forces on structural members are usually computed by means of Morison's equation. For a rigid vertical circular cylinder, whose velocities an accelerations may be considered to be negligible in the wave force calculation, wave force normal to the per unit length is described by Morison's equation $[3,4]$ :

$$
F=C_{D}|v| v+C_{M} \dot{v}=\frac{1}{2} \rho D C_{d}|v| v+\frac{-1}{4} \rho \pi D^{2} C_{m} \dot{v}=F_{v}+F_{a}
$$

where $v$ is the horizontal wave velocity component, $\dot{v}$ is the horizontal component of acceleration, $D$ is the diameter of circular cylinder, $C_{d}$ is the drag force coefficient, $C_{m}$ is the inertia force coefficient and $\rho$ is the water density. The first term denotes the drag, the second term specifies the inertia force.

When we assume that the wave motion is a Gaussian stationary process with zero mean, the velocity $v$ will be stationary Gaussian process too, with zero mean.

The drag force is important in the case $\frac{D}{\lambda} \leq 0.01$, where $\lambda$ being the wave length [6].

In the Morison's equation the drag force term is a nonlinear function of water particle velocity components while the inertia force term is a linear function of acceleration components. In order to overcome these difficulties the nonlinear drag force term must be linearized. Thus, we replace

$$
F_{v} \equiv \frac{1}{2} \rho D C_{d}|v| v \approx \frac{1}{2} \rho D C_{d} \gamma v
$$

The problem reduces to the linearized drag force (2.2) where the coefficient of linearization $\gamma$ is to be found from an optimal criterion. There are some criteria for determining the coefficient $\gamma$. The most extensively used criterion is the mean square error criterion which requires the mean square of error be minimum

$$
\left\langle e^{2}\right\rangle \rightarrow \min _{\gamma}
$$

where \langle\rangle denotes the expectation, and the replacement error $e$ is introduced

$$
e=|v| v-\gamma v
$$

Thus, from

$$
\frac{\partial}{\partial \gamma}\left\langle e^{2}\right\rangle=0
$$


it follows

$$
\gamma=\frac{\left\langle|v| v^{2}\right\rangle}{\left\langle v^{2}\right\rangle}=\sqrt{\frac{8}{\pi}} \sigma_{v} \approx 1.596 \sigma_{v}
$$

where $\sigma_{v}$ is the standard deviation of the process $v$.

So, the classical version of GEL, as described above, yields the coefficient of linearization $\gamma$ in (2.5). Rolfes and Dawson [10] carried out an experiment for measurement of the wave force of random waves acting on a Jacket structure. They observed that the linearized wave force based on the above mean square error criterion was larger than the experimental result about $17 \%$. Thus, one question is open : how the welknown mean square error criterion can be extended to improve the linearization of Morison's equation.

\section{Mean square criterion of error sample function}

An alternative approach to the problem is following. Let $F\left(e, a_{2}(\gamma), a_{3}(\gamma)\right.$, $\left.\ldots, a_{k}(\gamma)\right)$ be an arbitrary function of the error $e$ and parameters $a_{n}(\gamma)$ depending on the coefficient of linearization $\gamma,(n=2,3,4, \ldots, k)$. The function $F_{k}$ is called error sample function. Now, the mean square error criterion (2.5) can be extended to a mean square criterion of error sample function which requires that

$$
M_{k}=\left\langle F_{k}^{2}\left(e, a_{2}(\gamma), a_{3}(\gamma), \ldots, a_{k}(\gamma)\right)\right\rangle \rightarrow \min _{\gamma, a_{2}, a_{3}, \ldots, a_{k}}
$$

Thus, one gets

$$
\begin{aligned}
& \frac{\partial M_{k}}{\partial a_{n}}=\frac{\partial}{\partial a_{n}}\left\langle F_{k}^{2}\left(e, a_{2}(\gamma), a_{3}(\gamma), \ldots, a_{k}(\gamma)\right)\right\rangle=0 \\
& \frac{\partial M_{k}}{\partial \gamma}=\frac{\partial}{\partial \gamma}\left\langle F_{k}^{2}\left(e, a_{2}(\gamma), a_{3}(\gamma), \ldots, a_{k}(\gamma)\right)\right\rangle=0 .
\end{aligned}
$$

It is supposed that the error sample function is such that the system of $(k-1)$ equations (3.2) allows to define $k$ parameters $a_{2}, a_{3}, \ldots, a_{k}$ as functions of response mean square $\sigma_{v}$ and $\gamma$. Further, the latter can be definitely determined from (2.4) and the last equation of (3.2). It seems that the extended version of the classical mean square error criterion may contain many useful advantages. First of all, one can get a series of approximate response mean square by choosing different error sample functions $F_{k}\left(e, a_{2}(\gamma), a_{3}(\gamma), \ldots, a_{k}(\gamma)\right)$. This property of the extended criterion is very important from the point of view, of creating an approximate technique to solve non-linear stochastic problems.

The problem of choosing optimal error sample functions is open and waiting for it's a solution. In the following section a Hermite polynomial form of error sample functions will be proposed and investigated in detail for the Morison's equation. 


\section{t. Hermite polynomial error sample functions}

Consider a case in which the error sample functions are Hermite polynomials f error

$$
F_{1}=e ; \quad F_{k}=e-\sum_{i=2}^{k} a_{i} H_{2 i-1}(e), \quad k=2,3
$$

vhere $H_{n}(e)$ stands for the Hermite polynomials

$$
\begin{aligned}
& H_{0}(e)=1 \\
& H_{1}(e)=e \\
& H_{2}(e)=e^{2}-1 \\
& H_{3}(e)=e^{3}-3 e \\
& H_{4}(e)=e^{4}-6 e^{2}+3
\end{aligned}
$$

which satisfy the differentiation law

$$
\frac{d}{d e} H_{n}(e)=n H_{n-1}(e)
$$

and the recurrence relation

$$
H_{n+1}(e)=e H_{n}(e)-n H_{n-1}(e) .
$$

These polynomials are orthogonal on the interval $(-\infty,+\infty)$ with respect to the weght function $W(R)=\exp \left(-e^{2}\right)$. Thus

$$
\int_{-\infty}^{\infty} W(e) H_{n}(e) H_{m}(e) d e=0
$$

if $m$ is not equal to $n$.

From $(4: 1)$ one gets

$$
F_{k}^{2}=e^{2}-2 e \sum_{i=2}^{k} a_{i} H_{2 i-1}(e)+\sum_{i=2}^{k} \sum_{j=2}^{k} a_{i} a_{j} H_{2 i-1}(e) H_{2 j-1}(e) .
$$

The Hermite polynomials can be written as follows

$$
H_{n}(e)=\sum_{j=0}^{n} \mathbf{H}_{n, j} e^{j}
$$


where

$$
\left.\begin{array}{l}
\mathbf{H}_{0,0}=\mathbf{H}_{1,1}=1, \\
\mathbf{H}_{i+1, j+1}=\mathbf{H}_{i+1, j+1}+\mathbf{H}_{i, j} ; \quad j=0, i \\
\mathbf{H}_{i+1, j}=\mathbf{H}_{i+1, j}-i \mathbf{H}_{i-1, j} ; \quad j=0, i-1,
\end{array}\right\} i=1, n-1
$$

Denote $\cdot \mathbf{H}=\left[\mathbf{H}_{i, j}\right]$. In particular when $n=7$ one has

$$
\begin{array}{rcccccccc}
n & e^{0} & e^{1} & e^{2} & e^{3} & e^{4} & e^{5} & e^{6} & e^{7} \\
0 & 1 & & & & & & & \\
1 & & 1 & & & & & & \\
\mathbf{H} & -1 & & 1 & & & & & \\
3 & & -3 & & 1 & & & & \\
4 & 3 & & -6 & & 1 & & & \\
5 & & 15 & & -10 & & 1 & & \\
6 & -15 & & 45 & & -15 & & 1 & \\
7 & & -105 & & 105 & & -21 & & 1
\end{array}
$$

Thus, one gets

$$
\begin{aligned}
& H_{2 i-1}(e)=\sum_{k_{1}=0}^{2 i-1} \mathbf{H}_{2 i-1, p} e^{p} \\
& H_{2 i-1}(e) H_{2 j-1}(e)=\sum_{p=0}^{2 i-1} \sum_{q=0}^{2 j-1} \mathbf{H}_{2 i-1, p} \mathbf{H}_{2 j-1, p} e^{(p+q)}, \\
& \left\langle F_{k}^{2}\right\rangle=\left\langle e^{2}\right\rangle-2 \sum_{i=2}^{k}\left(a_{i} \sum_{p=0}^{2 i-1} \mathbf{H}_{2 i-1, p}\left\langle e^{(p+1)}\right\rangle\right) \\
& \quad+\sum_{i=2}^{k} \sum_{j=2}^{k}\left(a_{i} a_{j} \sum_{p=0}^{2 i-1} \sum_{q=0}^{2 j-1} \mathbf{H}_{2 i-1, p} \mathbf{H}_{2 j-1, p}\left\langle e^{(p+q)}\right\rangle\right\} \\
& \frac{\partial\left\langle F_{k}^{2}\right\rangle}{\partial a_{i}}=0 .
\end{aligned}
$$

Since the mean square $\left\langle F_{k}^{2}\right\rangle$ is definitely positive, the system of linear equations (4.10) gives a unique solution for unknown parameters $a_{j}$ in the form

$$
\begin{aligned}
& \mathbf{a}=\mathbf{A}^{-1} \mathbf{p} \\
& \mathbf{A}_{i, j}=2 H_{2 i-1}(e) H_{2 j-1}(e) \\
& \mathbf{p}_{i}=2 e H_{2 i-1}(e) .
\end{aligned}
$$


On the other hand, one gets from (2.4) the relation

$$
\left\langle e^{2 n}\right\rangle=\left\langle(|v| v-\gamma v)^{2 n}\right\rangle ; \quad n=1,2,3, \ldots
$$

which shows that error even moments $\left\langle e^{2 n}\right\rangle$ can be expressed in terms of $\sigma_{v}$

$$
e^{2 n}=(|v| v-\gamma v)^{2 n}=\sum_{i=0}^{2 n} C_{2 n}^{i}(-1)^{i}(|v| v)^{2 n-i} v^{i} \gamma^{i}
$$

Denote:

$$
x=\frac{\gamma}{\sigma_{v}}
$$

for even $i: i=2 j$

$$
\begin{aligned}
\sum_{i=0}^{2 n} C_{2 n}^{i}(-1)^{i}(|v| v)^{2 n-i} v^{i} \gamma^{i} & =\sum_{j=0}^{n} C_{2 n}^{2 j}\left(v^{2}\right)^{2 n-2 j} v^{2 j} \gamma^{2 j} \\
& =v^{4 n} \sum_{j=0}^{n} C_{2 n}^{2 j} v^{4 n-2 i} \gamma^{2 j}
\end{aligned}
$$

for odd $i: i=2 j+1$

$$
\begin{aligned}
\sum_{i=0}^{2 n} C_{2 n}^{i}(-1)^{i}(|v| v)^{2 n-1} v^{i} \gamma^{i} & =-\sum_{j=0}^{n-1} C_{2 n}^{2 j+1}|v|^{2 n-(2 j+1)} v^{2 n} \gamma^{2 j+1} \\
& =-\sum_{j=0}^{n-1} C_{2 n}^{2 j+1}|v| v^{4 n-2 j-2} \gamma^{2 j+1}
\end{aligned}
$$

Since. $v$ is a Gaussian process one gets:

$$
\begin{aligned}
\left\langle|v| v^{2 k}\right\rangle=2 \int_{0}^{+\infty} v^{2 k+1} \frac{1}{\sqrt{2 \pi} \sigma_{v}} \exp \left(\frac{-v^{2}}{2 \sigma_{v}^{2}}\right) d v=(2 k) ! ! \sigma_{v}^{2 k+1} \sqrt{\frac{2}{\pi}}, \\
\left\langle e^{2 n}\right\rangle=\left\langle v^{4 n}\right\rangle \sum_{j=0}^{n} C_{2 n}^{2 j}\left\langle v^{4 n-2 j}\right\rangle \gamma^{2 j}-\sum_{j=0}^{n-1} C_{2 n}^{2 j+1}(4 n-2 j-2) ! ! \sigma_{v}^{4 n-(2 j+1)} \gamma^{2 j+1} \sqrt{\frac{2}{\pi}} \\
=\sigma_{\nu}^{4 n}\left[\sum_{j=0}^{n} C_{2 n}^{2 j}(4 n-2 j-1) ! ! x^{2 j}-\sum_{j=0}^{n-1} C_{2 n}^{2 j+1}(4 n-2 j-2) ! ! x^{2 j+1} \sqrt{\frac{2}{\pi}}\right],
\end{aligned}
$$


where it is denoted

$$
\begin{aligned}
& (2 k+1) ! !=1.3 .5 \ldots(2 k+1), \\
& (2 k) ! !=2.4 .6 \ldots(2 k) .
\end{aligned}
$$

So, the function $\left\langle F_{k}^{2}\right\rangle$ depends only on $\gamma$ and $\sigma_{v}$. It can be shown that now the function $\mathrm{M}$ can be expressed in the form

$$
M_{k}=\left\langle F_{k}^{2}\right\rangle=\sigma_{v}^{4} N_{k}(x),
$$

where $N_{k}(x)$ is a known polynomial of $x$.

Denote by $\gamma_{k}$ the corresponding solution obtained from the conditions $\left\langle F_{k}^{2}\right\rangle \rightarrow$ min. It might be expected that the approximate solution $\gamma_{k}$ would approach to the exact one, respectively, as one has clearly the following series of inequalities

$$
\min _{\gamma}\left\langle e^{2}\right\rangle \geq \min _{\gamma, a_{1}}\left\langle F_{2}^{2}\right\rangle \geq \min _{\gamma, a_{\dot{1}}, a_{2}}\left\langle F_{3}^{2}\right\rangle \geq \cdots \geq \min _{\gamma, a_{1}, a_{2}, \ldots, a_{k}}\left\langle F_{k}^{2}\right\rangle .
$$

Thus, one can write the following numerical procedure for minimizing $N_{k}(x)$ :

1. Input data : $k, x_{0}, x_{1}, n_{x}$

2. Computer matrix $\mathrm{H}$ by using (4.6) for $n=2 k$

3. Enter loop covering all $n_{x}$ points $x$ from $x_{0}$ to $x_{1}$

3.1. Computer matrix $A$ and vector $p$ by using (4.12)

3.2. Computer vector a by using (4.11)

3.3. Computer $M_{k}$ and $N_{k}(x)$ by using (4.9), (4.19), (4.21)

3.4. Determine Min $N_{k}(x)$ and $x_{\min }$.

End loop.

\section{First five approximate solutions}

The approximate solution $\gamma_{k}$ is called $k^{t h}$ approximate one. In this section the equations used to determine the coefficient of linearization corresponding to five first approximate solutions are derived in the explicit form. Obviously, the first approximate solution $\gamma_{1}$ is identical to the one obtained from the classical mean square error criterion since $F_{1}=e$. First five approximate solutions for $\gamma_{1}$

\begin{tabular}{|c|c|c|}
\hline$k^{\text {th }}$ Approximation & $x_{k}=\frac{\gamma_{k}}{\sigma_{v}}$ & Error $(\%)$ \\
\hline 1 & 1.596 & 17.0 \\
\hline 2 & 1.482 & 8.6 \\
\hline 3 & 1.442 & 5.7 \\
\hline 4 & 1.402 & 2.8 \\
\hline 5 & 1.381 & 1.2 \\
\hline
\end{tabular}
are given in Tab.1, while the corresponding graphics of $N_{k}(x)$ are given in Figs $1-4$.

Tab. 1. Five first approximate $\gamma_{k}$ 
From calculations, we can do the following comparison: Suppose the first approximation for the nonlinear drag force term in Morison's equation, $0.5 \rho D C_{d} \times$ $\left(1.596 \sigma_{v}\right) v$, is larger than the experimental result about $17 \%$ as observed in [10]. Thus, one obtains that the fifth approximation, $0.5 \rho D C_{d}\left(1.381 \sigma_{v}\right) v$, will be larger than the experimental result only $1.2 \%$.

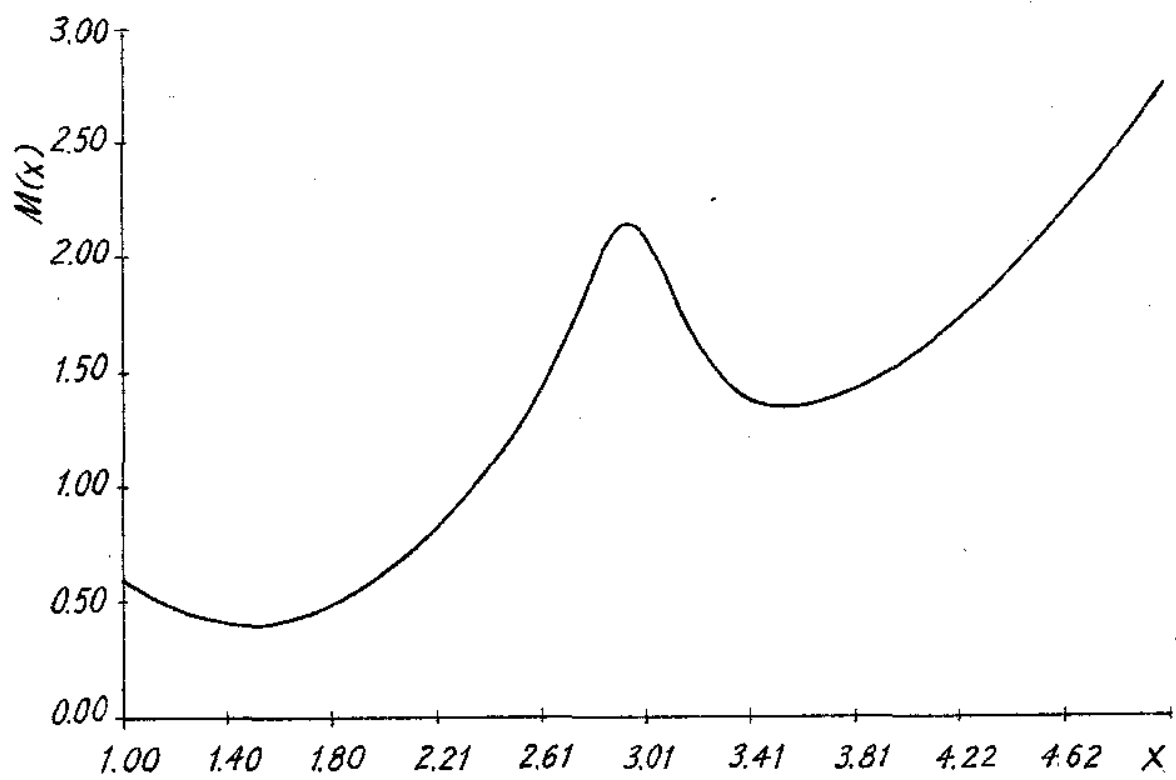

Fig. 1. Graphic of the function $N_{2}(x)=\frac{1}{\sigma_{v}^{4}}\left\langle\left(e-a_{2} H_{3}(e)\right)^{2}\right\rangle$ for the second approximation

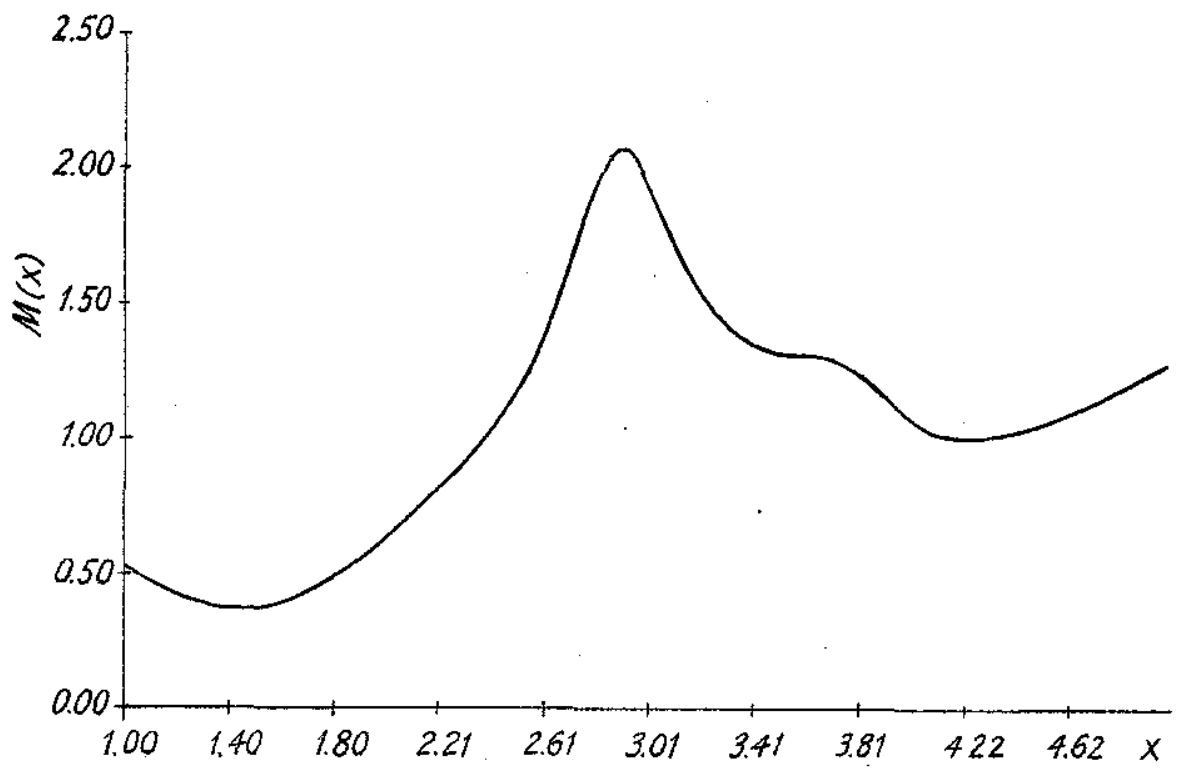

Fig. 2. Graphic of the function $N_{3}(x)=\frac{1}{\sigma_{n}^{4}}\left\langle\left(e-a_{2} H_{3}(e)-a_{3} H_{5}(e)\right)^{2}\right\rangle$ for the third approximation 


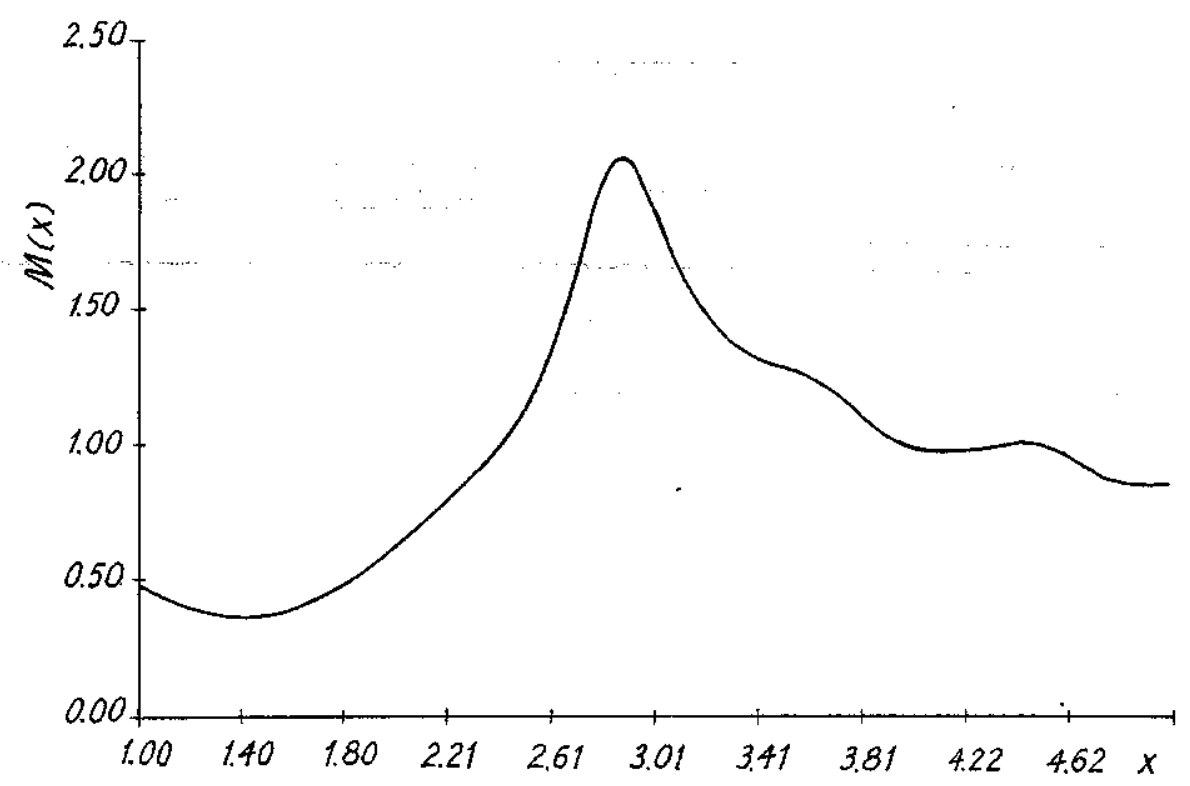

Fig. 3. Graphic of the function $N_{4}(x)=\frac{1}{\sigma_{v}^{4}}\left\langle\left(e-a_{2} H_{3}(e)-a_{3} H_{5}(e)-a_{4} H_{7}(e)\right)^{2}\right\rangle$ for the fourth approximation

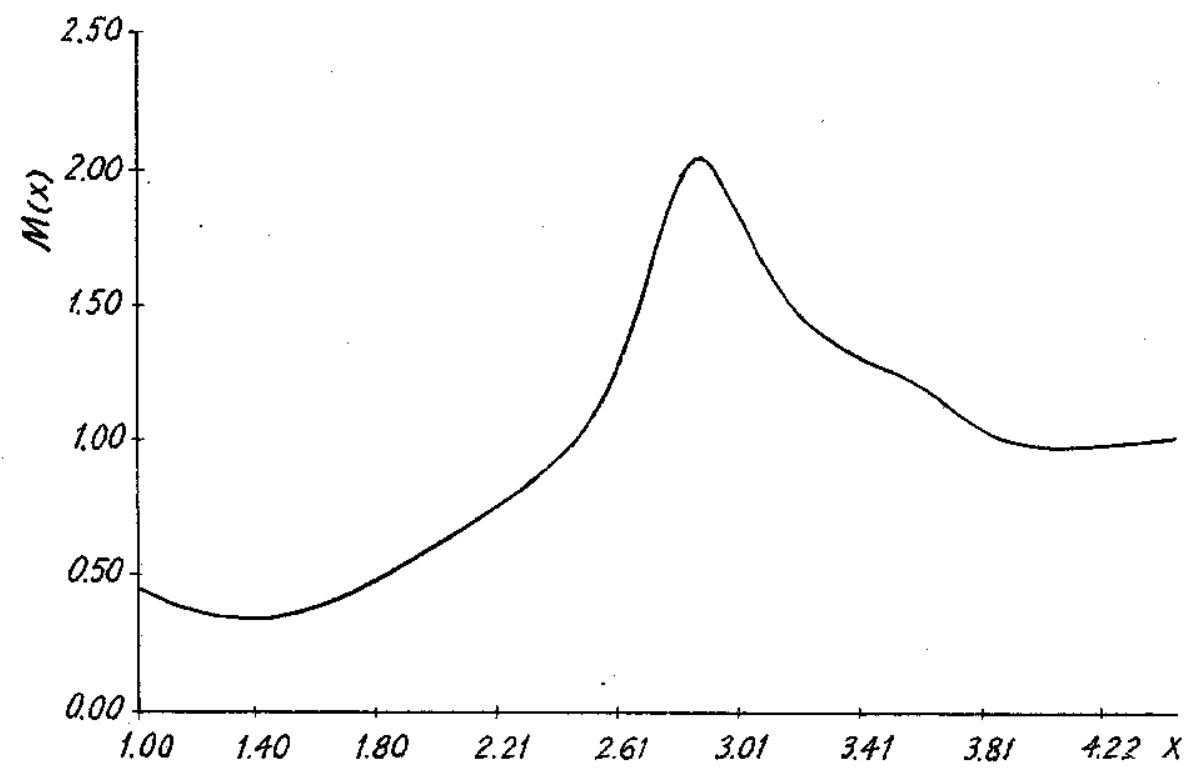

Fig. 4. Graphic of the function

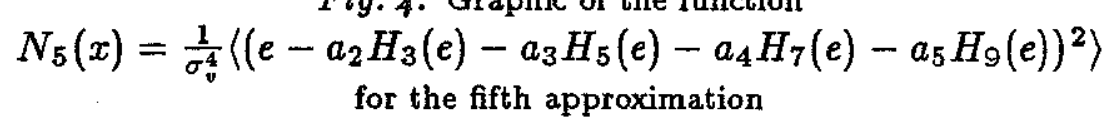

\section{Conclusions}

The main question inherent in Gaussian linearization is how the coefficients of the linearized equation are found. Instead of the well-known mean square error criterion, a mean square criterion of error sample function based on Hermite poly- 
nomials has been proposed to linearize the drag force in the Morison's equation. An important property of this extended criterion is that it gives a possibility to obtain a series of approximation-including the conventional one as the first approximation. Further first five corresponding approximations are investigated in detail. Application to the drag force in the Morison's equation shows the reducing the error between theoretical and experimental result from $17 \%$ to $1.2 \%$.

This paper is completed with financial support of the Council for Natural Sciences of Vietnam.

\section{References}

1. Anh N. D., Schiehlen W. New criterion for Gaussian equivalent linearization, European J. Mechanics/Solid, v. 16, No6, 1997, 1025-1039.

2. Atalik T. S., Utku S. Stochastic linearization of multi-degree-of-freedom nonlinear system, J. Earth. Eng. Struct. Dynamics, 4, 1976, 441-420.

3. Casciati F., Faravelli L. Equivalent linearization in non-linear random vibration problems, Proc. Int. Conf. On Vibration Problems in Eng., Xian, China, 1986, 986-991.

4. Caughey T. K. Response of a non-linear string to random loading, J. Appl. Mech. 26, 1959, 341-344.

5. Dawson T. Offshore structural Engineering, Prentice-Hall Inc., New Jersey 1983.

6. Haritos. Hydrodynamic damping of flexibly supported cylinders in waves, Proc. of Symp. on Hydrodynamics in Ocean Eng-ing, NIT, 1981, 217-238.

7. Hu. W. Koterayama. A study on random wave force coefficients using numerical simulation of flow field. Proc. III IOPEC, Singapore 1993.

8. Lee Y. J. Lan. Wave forces on vertical circular cylinders, Proc. III IOPEC, Singapore, 1993.

9. Roberts J. B.; Spanos P. D. Random vibration and stochastic linearization, John Wiley and Sons, 1990.

10. Rolfes J. B., Dawson T. Forces on a large scale offshore test structure in random waves, Proc. HOE, NIT, 1981, 509-530.

Received June 20, 1998

TUYẾN TÍNH HÓA TƯONG ĐUƠNG LỰC VẬN TỐC TRONG PHUƠNG TRINNH MORISON BẰNG ĐA THỨC HERMITE CƯA HÀM ĐỘ LỆCH

Một tiêu chuẩn bình phương trung bình mới được đề nghị trên cơ sớ sứ dụng đa thức Hermite của hàm độ lệch để xác định các hệ số tuyến tính hóa tương đương đối với các quá trình ngẫu nhiên Gauss với trung bình bằng 0 . Áp dụng cho phương trình Morison đối với lực sóng biển nhận được kết quả tốt hơn so với phương pháp tuyến tính hóa tương đương kinh điển. 